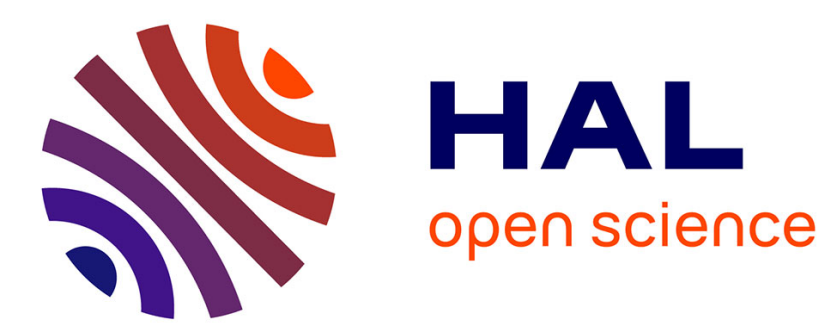

\title{
TRANSIENT GRATING EXPERIMENTS IN PERCOLATION FRACTALS
}

P. Evesque, J. Duran, A. Bourdon

\section{To cite this version:}

P. Evesque, J. Duran, A. Bourdon. TRANSIENT GRATING EXPERIMENTS IN PER-

COLATION FRACTALS. Journal de Physique Colloques, 1985, 46 (C7), pp.C7-45-C7-49. 10.1051/jphyscol:1985709 . jpa-00224957

\section{HAL Id: jpa-00224957 https://hal.science/jpa-00224957}

Submitted on 1 Jan 1985

HAL is a multi-disciplinary open access archive for the deposit and dissemination of scientific research documents, whether they are published or not. The documents may come from teaching and research institutions in France or abroad, or from public or private research centers.
L'archive ouverte pluridisciplinaire HAL, est destinée au dépôt et à la diffusion de documents scientifiques de niveau recherche, publiés ou non, émanant des établissements d'enseignement et de recherche français ou étrangers, des laboratoires publics ou privés. 


\title{
TRANSIENT GRATING EXPERIMENTS IN PERCOLATION FRACTALS
}

\author{
P. Evesque, J. Duran and A. Bourdon \\ Laboratoire d'Optique de la Matière Condensée, UA 800 du C.N.R.S., Tour 13, \\ 4 place Jussieu, 75230 Paris Cedex 05, France
}

\begin{abstract}
Résumé
Nous prédisons les résultats des expériences de réseau transitoire sur un système désordonné en utilisant le concept de l'espace fractal et la théorie de la percolation. Nous montrons, en particulier que le résultat de ces expériences dépend du rapport entre l'interfrange du réseau transitoire et de la longueur de corrélation du désordre. Ainsi, une expérience effectuée avec un interfrange plus petit que la longueur de corrélation reflétera les propriétés de l'espace fractal de la diffusion à travers une dépendance anormale du temps de déclin en fonction de l'interfrange du réseau.
\end{abstract}

\begin{abstract}
Using both the concept of the fractal space and the percolation theory in the critical region we predict the behaviour of the transient grating experiments in the two extreme cases when the interfringe is much larger or smaller than the correlation length of the disorder. In particular we show that when performed in the small interfringe limit, the result of the se experiments will directly reflect the properties of the fractal space of the diffusion through an anomalous interfringe dependence of the decay time of the transient grating.
\end{abstract}

\section{I - INTRODUCTION}

It has been demonstrated recently, both theoretically and experimentally, that several problems arising in disordered materials could by fruitfully tackled through a convenient mapping of the disordered material on a percolation model $/ 2,5,6 /$. The main advantage of such a procedure consists in allowing to describe most of the dynamic properties using the spectral exponent $\widetilde{d}$ defined by Alexander and Orbach $/ 1 /$. As pointed out by these authors, this exponent controls as well the density of states of the infinite cluster at the percolation threshold as the kinetics of a random walk process on this cluster. Consequently as far as the characteris tic length scale of the experiment will be smaller than the correlation length of the disorder, any dynamic property of a random system will be governed by the spectral exponent $\tilde{d}$. For larger experimental length scales, on the other hand, de Gennes /4/ and Gefen et al. /8/ predicted a classical behaviour. 
From the experimental standpoint, the so-called transient grating technique has been proved repeatedly to provide a convenient and direct insight in the diffusion properties of the investigated materials $/ 7,3 /$. Basically it consists in inter secting two coherent and simultaneous laser pulses at an angle $\theta$ in a sample. In turn the resulting sinusoidal interference pattern diffracts a third delayed probe beam which provides an estimate of the residual contrast of the transient grating at any desired moment after the exciting pulse. As will be reminded shortly afterwards, it is quite intuitively understood that any diffusion process will tend to fill in the zeros of the initially printed grating. Also the time dependence of the intensity of the diffracted beam will largely depend on the interfringe $\Omega$ of the grating which turns out to play the role of the characteristic length of the measurement.

This work is aimed at predicting the extinction characteristic of the first order of the diffracted probe beam in a transient grating experiment on a disordered system which can be mapped on a percolation model.

\section{II - TRANSIENT GRATING EXPERIMENTS ON A PERCOLATION FRACTAL MODEL}

A complete analytical solution to the transient grating experiment in an homogeneous diffusing medium has been put forth by Salcedo et al. /10/. However, keeping in mind that our purpose is to relate disorder and transient grating experiments, we propose an alternative demonstration to the basic result of this study. We consider the characteristic time of the extinction of the first order diffracted probe beam which corresponds to the time needed by a random walking excitation to travel on a length scale of $\Lambda$. If $\xi$ stands for the length of an elementary jump, the number of necessary steps will be proportional to $\Lambda / \xi$ and this quantity is related to the time $T$ needed by the random walker to execute this trip via a clas sical equation given by Alexander and Orbach / 1 /:

$$
\frac{\Lambda}{\xi} \sim\left(\frac{T}{T_{0}}\right)^{1 / 2}
$$

Where $T_{0}$ is the elementary jump time. So that we find :

$$
\mathrm{T} \sim \mathrm{T}_{0} \Lambda^{2} / \xi^{2}
$$

Let us turn now to the consideration of a transient grating experiment in a medium which exhibits bond or (and) site disorder. In such a system the jumps will be either possible or not, depending on the pairs of sites considered so that it is expected that the problem will not be controled by the single $\Lambda$ parameter but also by a correlation length of disorder $\boldsymbol{\xi}$. This parameter $\boldsymbol{\xi}$ may either describe the characteristic length of the disorder in the infinite cluster or the length of the largest likely finite clusters. Considering the nature of the problem, we are now led to introduce two limit cases following an approach similar to the one dimensional case /3/. As we show in the following the solution of the problem may be derived easily in what we call the small and the large interfringe limits which are naturally defined as :

$$
\left\{\begin{array}{l}
\text { small interfringe limit }: \Lambda \ll \xi \\
\text { large interfringe limit }: \Lambda \gg \xi
\end{array}\right.
$$

In the following we shall examin successively these two limit cases. 


\section{A) Large interfringe limit}

In this paragraph we will deal essentially with the diffusion of the excitation on the infinite cluster which implies that the system is above the percolation threshold. Be $c_{c}$ the critical concentration of the percolation process and $c$ the concentration which corresponds to the real system when mapping it on percolation model. We define $\Delta \mathrm{c}$ as the relative deviation of the critical concentration according to :

$$
\Delta c=\frac{c-c_{c}}{c_{c}}
$$

The percolation theory predicts that the probability $\mathrm{P}_{\infty}$ that a given site pertain to the infinite cluster is:

$$
\begin{array}{ll}
P_{\infty}=0 & \text { for } \Delta c<0 \\
P_{\infty} \sim \Delta c^{\beta} & \text { for } 0<\Delta c \ll 1
\end{array}
$$

Where $\beta$ is the classical critical exponent of the percolation theory as defined by Stauffer $/ 11 /$.

We now turn to the analysis of the concentration dependence of the decay time of the diffracted probe beam. According to a procedure reported by de Gennes $/ 4 /$ we note that the infinite cluster exhibits a local fractal structure. The fractal and spectral dimensions of this peculiar space are respectively $D$ and $\widetilde{d} / 9 /$ at least when the system is considered on a small length scale $l(l<\xi)$. It has been shown that, under these circumstances, the mean number of distinct visited sites between the origin of the time and time $t$, is given by $/ 4 /$ :

$$
N(t) \sim\left(\frac{\ell}{a}\right)^{D} \sim\left(\frac{t}{c}\right)^{\tilde{d} / 2}
$$

where a and $\tau$ stand respectively for the length and the time corresponding to an elementary jump of the excitation. As has been shown by Alexander and Orbach $/ 1 /$, the spectral dimension $\widetilde{d}$ lies very near $4 / 3$ whatever the dimension of the space of the percolation is.

As we mentioned previously, the diffusion process must turn out to a classical one as soon as the length scale becomes larger than the correlation length of the disorder $\xi$. If this occurs, we expect that the system will be correctly described by Eq. (1). If now we want to achieve a continuity 'relationship between Eq. (1) and Eq. (4), that is to say between the macroscopic and the microscopic regimes, we are compelled to express the kinetic of the diffusion under the macroscopic regime through:

$$
\frac{l}{\xi} \sim\left(\frac{t}{\mathrm{~T}_{0}}\right)^{1 / 2}
$$

Where $l$ is much larger than $\xi$ and $T_{0}$ is a parameter which must be adjusted in order to get the continuity between the microscopic and the macroscopic regimes. This can be done easily by considering Eq. (4) at the point when $l$ turns out to be exactly equal to the correlation length of disorder $\xi ;$ then $t$ is equal to $T_{0}$ and we get from Eq. (4) :

$$
\left(\frac{\xi}{a}\right)^{\mathrm{D}} \sim\left(\frac{T_{0}}{\varepsilon}\right)^{\tilde{\mathrm{d}} / 2}
$$

If we realize that the characteristic time $T$ of the extinction of the first order diffracted probe beam corresponds to a diffusion length equal to an interfringe $\Lambda$, we calculate $\mathrm{T}$ by replacing $\ell$ by $\Lambda$ in Eq. (5a) and plugging the $T_{0}$ value 
extracted from ( $5 b)$, so that we get :

$$
\mathrm{T}=\tau\left(\frac{\xi}{\mathrm{a}}\right)^{2 \mathrm{D} / \tilde{\mathrm{d}}^{-2}}\left(\frac{\Lambda}{\mathrm{a}}\right)^{2}
$$

Stauffer /11/ has reported that $\xi$ can be expressed as function of the concentration $\Delta c$ via the critical relationship :

$$
\left(\frac{\xi}{a}\right) \sim \Delta c^{-\nu}
$$

Which finally leads to the value of the transient grating decay time in the large interfringe limit

$$
T \sim \tau\left(\frac{\Lambda}{a}\right)^{2}(\Delta c)^{-2 \nu(D / \tilde{d}-1)} \text { with } \Delta c>0
$$

Moreover as the kinetics of the random walk process is quite classical in this macroscopic regime $(\ell \gg \xi)$ the decay law of the first order diffracted beam should be simply given by :

$$
I(t)-I_{\infty}=I_{0} C e^{-2 t / T}
$$

\section{B) Small interfringe limit}

As mentioned earlier, this case corresponds to the existence of large finite clusters the length of which being larger than the interfringe $\Lambda$ (Fig. 1 ). As a matter of fact, the complete calculation of the time dependence of the transient grating signal turns out to be quite intricate in this critical regime, so that we will use various convenient simplifications in the present work. In particular, we will consider that only those clusters exhibiting a spatial expansion larger that the interfringe distance will contribute to the transient grating decay. Another important point concerns the fact that the large clusters will, generally, covers more than a single interfringe so that we can consider here that the equilibrium which will be reached on these large clusters will be independent of their size and similar to this one reached on the infinite cluster.

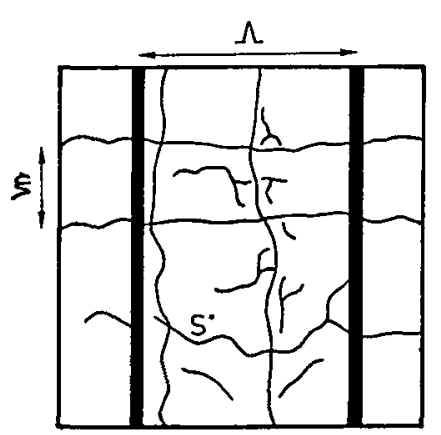

$\Lambda \gg \xi \quad(b)$

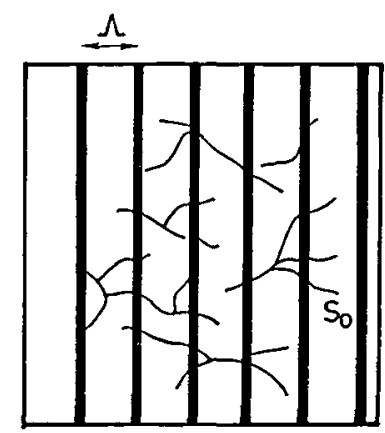

$\Lambda \ll \xi$

(a)

\section{Figure 1}

(a) Small interfringe limit $(\xi \gg \Lambda),\left(S^{*}=\xi^{D}\right)$. Finite clusters of size larger than $\Lambda^{D}$ exist.

(b) Large interfringe limit $(\xi \ll \Lambda),\left(\mathrm{S}^{*}=\xi^{\mathrm{D}}\right)$. Only finite clusters of size much smaller than $\Lambda$ exist in the sample; the diffusion can then only occur in the infinite cluster if it exists. 
It results from the present analysis that the time dependence of the diffracted probe beam will be mostly governed by a typical class of clusters with size $\Lambda$. The characteristic time of the decay $\mathrm{T}$ will be estimated by calculating the time needed by a random walker to explore one of the se typical clusters.

As each finite cluster is mapped on a fractal space with fractal and spectral dimensions respectively $D$ and $\tilde{\mathrm{d}}$. the kinetics of the diffusion process is given by Eq. (4), so that we get the characteristic time $T$ from :

$$
\left(\frac{\Lambda}{a}\right)^{D} \sim\left(\frac{T}{\tau}\right)^{\tilde{d} / 2}
$$

which gives :

$$
T \sim \tau\left(\frac{\Lambda}{a}\right)^{2 D / \tilde{d}}
$$

\section{CONCLUSION}

As shown in the preceding paragraph, the study of the diffusion in a disordered medium through a transient grating experiment involves two different regimes. The first one, which corresponds to a macroscopic regime will occur when the interfringe $\Lambda$ is larger than the correlation length of the disorder $\xi$. In this case the characteristic decay time of the grating will scale through the classical law $\left(T \sim \Lambda^{2}\right)$.

Now, if the interfringe is reduced till it becomes smaller than the correlation length of the disorder $\xi$, one should expect a cross-over between the previously mentioned macroscopic regime through a critical one. This last regime is characterized by a decay time of the diffracted probe beam which will scale unclassically with the interfringe distance.

\section{Achnowledgements}

The authors are indebted to Dr. Daoud and Prof, P. G. de Gennes for stimulating discussions.

\section{$\underline{\text { References }}$}

11/ Alexander, S. and Orbach, S, J. de Physique Lettres (Paris) 43 (1982) L-625

/2/ Argyrakis, P. and Kopelman, R., J.Chem. Phys. 66 (1977) 3301

/3/ Bourdon, A., Duran, J., Pellé, F. and de Viry, D., Phys. Rev. B 30 (1984) 7105

/4/ de Gennes, P. G. , C. R. Acad. Sc. Paris 296 (1983) 881

/5/ Evesque, P., J. de Physique (Paris) $4 \underline{4}$ (1983) 1217

/6/ Evesque, P. and Duran, J., J. Chem. Phys. 80 (1984) 3016

/7/ Fayer, M.D., Picosecond Phenomena III, Springer series in Chemical Physics 23, ed. F. Shäfer Springer Verlag Berlin 1982

/8/ Gefen, Y., Aharony, A., Alexander, S., Phys, Rev. Lett. 50 (1983) 77

19/ Rammal, R. and Toulouse, G., J. de Physique Lettres (Paris) 44 (1983) L13

/10/Salcedo, J.R., Siegman, A.E., Dlott, D. D, and Fayer, M. D., Phys, Rev. Lett. 41 (1978) 131

/11/Stauffer, D., Phys, Rev, $\underline{54}$ (1979) 1 